\title{
Article
}

\section{COVID-19 Prevalence among Czech Dentists}

\author{
Jan Schmidt $^{1}$, , Vojtech Perina ${ }^{2, *}$, Jana Treglerova ${ }^{2}$, Nela Pilbauerova ${ }^{1}$ (D) Jakub Suchanek ${ }^{1}$ (D) \\ and Roman Smucler ${ }^{3}$
}

1 Department of Dentistry, Charles University, Faculty of Medicine in Hradec Kralove and University Hospital Hradec Kralove, 50005 Hradec Kralove, Czech Republic; Jan.Schmidt@lfhk.cuni.cz (J.S.); Nela.Pilbauerova@lfhk.cuni.cz (N.P.); SuchanekJ@lfhk.cuni.cz (J.S.)

2 Department of Oral and Maxillofacial Surgery, Masaryk University, Faculty of Medicine and University Hospital Brno, 62500 Brno, Czech Republic; treglerova.jana@fnbrno.cz

3 Czech Dental Chamber, Slavojova 270/22, 12800 Prague, Czech Republic; Smucler@dent.cz

* Correspondence: Perina.Vojtech@fnbrno.cz

Citation: Schmidt, J.; Perina, V.; Treglerova, J.; Pilbauerova, N.; Suchanek, J.; Smucler, R. COVID-19 Prevalence among Czech Dentists. Int. J. Environ. Res. Public Health 2021, 18, 12488. https://doi.org/10.3390/ ijerph182312488

Academic Editor: Dirga

Kumar Lamichhane

Received: 2 November 2021

Accepted: 24 November 2021

Published: 27 November 2021

Publisher's Note: MDPI stays neutral with regard to jurisdictional claims in published maps and institutional affiliations.

Copyright: (C) 2021 by the authors. Licensee MDPI, Basel, Switzerland. This article is an open access article distributed under the terms and conditions of the Creative Commons Attribution (CC BY) license (https:// creativecommons.org/licenses/by/ $4.0 /)$.

\begin{abstract}
This work evaluates the prevalence of coronavirus disease (COVID-19), a viral infection caused by severe acute respiratory syndrome coronavirus 2 (SARS-CoV-2), among members of the Czech Dental Chamber. The assessment was based on an online questionnaire filled out by 2716 participants, representing $24.3 \%$ of all chamber members. Overall, $25.4 \%$ of the participants admitted they were diagnosed with COVID-19 by 30 June 2021, with no statistical differences between the sexes. While in the age groups under 50 the reported prevalence was around $30 \%$, with increasing age, it gradually decreased to $15.2 \%$ in the group over 70 years. The work environment was identified as a place of contagion by $38.4 \%$ of the respondents. The total COVID-19 PCR-verified positivity was $13.9 \%$, revealing a statistically lower prevalence $(p=0.0180)$ compared with the Czech general population, in which the COVID-19 PCR-verified positivity was $\sim 15.6 \%$ (fourth highest rank in the world). The total infection-hospitalization ratio (IHR) was $2.8 \%$, and the median age group of hospitalized individuals was 60-70 years. For respondents older than 60 years, the IHR was $8.7 \%$, and for those under 40 years, it was $0 \%$. Of the respondents, $37.7 \%$ admitted that another team member was diagnosed with COVID-19, of which the most frequently mentioned profession was a nurse/dental assistant (81.2\%). The results indicate that although the dentist profession is associated with a high occupational risk of SARS-CoV-2 infection, well-chosen antiepidemic measures adopted by dental professionals may outweigh it.
\end{abstract}

Keywords: COVID-19; SARS-CoV-2; prevalence; dentistry; pandemic; dentist; occupational health; infection

\section{Introduction}

Coronavirus disease (COVID-19) is a viral infection caused by the newly isolated severe acute respiratory syndrome coronavirus 2 (SARS-CoV-2). The standard clinical features are of a wide flulike spectrum, including fatigue, taste and smell loss, cough, headache, or fever. However, in some patients, it can lead to a more severe form, including breathing difficulties, respiratory failure, or acute inflammatory response, which could be fatal [1,2]. The rapid spread of SARS-CoV-2 is mainly due to the type of its transmission from person to person via respiratory droplets or mucosal contact or less often by contact with fomites [3-5]. The first official case of SARS-CoV-2 was reported in Wuhan City, Hubei Province, China, in December 2019 [6]. Due to its global spread, it soon became a worldwide health threat broadly affecting human society and leading the World Health Organization to classify COVID-19 as a pandemic disease as of 11 March 2020 [7].

The first cases of COVID-19 were recorded in the Czech Republic at the beginning of March 2020. The Czech government quickly issued a number of antiepidemic measures, which made the virus spread very limited. At the end of August 2020, the cumulative numbers of COVID-19 PCR-verified cases and total deaths per 100,000 people were 230 
and 4, respectively [8,9]. However, since September 2020, the number of infected patients has risen sharply. During the autumn of 2020 and the spring of 2021, the Czech Republic was one of the countries most affected by COVID-19. As of the reference period of this study (i.e., 30 June 2021), the Czech Republic had 15,546 cumulatively PCR-verified infected per 100,000 people, which was the fourth highest number in the world [10]. On the same date, the number of total deaths related to COVID-19 per 100,000 people was 283, which was the fourth highest number in the world [11].

The transmission of SARS-CoV-2 is mainly via droplets, and in areas where there is a great fluctuation and accumulation of individuals, the spread of the disease is heightened. This also applies to medical facilities, making healthcare professionals vulnerable to COVID19 , with a special risk for those whose work is associated with mucus and saliva droplets. This is especially true for dental professionals. The dentist's work is associated with close contact with many people and producing a large amount of aerosol containing the patient's saliva and mucus droplets. Due to the high speed of dental rotary instruments, the aerosol swirls at a high speed to a distance of several meters from the source. Thus, the work environment of dentists is particularly risky, and dentists are one of the highly vulnerable groups [12].

During the COVID-19 pandemic, general healthcare was suppressed in the Czech Republic. However, a survey performed among members of the Czech Dental Chamber revealed that Czech dentists worked even throughout the pandemic [13]. During the spring of 2020, in the Czech Republic also called the "first wave" of COVID-19, more than $90 \%$ of the participating dentists replied that their practices were open. During the period from autumn 2020 to spring 2021, also called the "second wave" of COVID-19, more than $96 \%$ of them replied their practices remained open. From those who closed their practices during the period from March 2020 to March 2021, only less than 10\% reported that the closure was longer than 4 weeks. The data showed that Czech dentistry remained very operational during the whole pandemic. This approach was rare on a European and global scale [13]. Such conditions make Czech dentists a unique study group to assess the impact of COVID-19 on dental professionals as it minimizes the bias resulting from their workplace.

Based on the combination of these three factors-high national prevalence, a significant risk of infection due to work settings, and high workload during pandemics-Czech dentists form a unique group with a presumption of high COVID-19 prevalence. At the same time, it could be assumed that dentists will be more affected by COVID-19 than the Czech general population due to the work environment. Furthermore, as Czech dentists remained more operative during the pandemic than their counterparts in other countries, it can be assumed that the regional impact of COVID-19 on this professional group was greater. However, these assumptions are hypotheses only and have not yet been addressed in any study.

On the other hand, Czech dentists were aware of these risks, and in order to maintain high operability, they adopted strict antiepidemic measures, such as an anamnestic questionnaire for each patient, regular testing of dental team members, planning a daily schedule to minimize patient accumulation in dental practices, rubber dam use, barrier precautions, minimizing aerosol spread, or establishing dental centers for the treatment of COVID-19-positive patients. These measures were aimed at minimizing the risk of transmission from patients to staff and vice versa, between staff, and between patients. The Czech Dental Chamber was one of the first dental chambers in Europe to issue antiepidemic recommendations for its members, and ordinary members of the chamber were also very proactive in this regard. These thorough measures could significantly reduce the risk of COVID-19 transmission in dental practices. However, so far, there are no data available to confirm this assumption.

To reflect the need to obtain statistically relevant quantifying data, the Czech Dental Chamber decided to conduct a survey among its members, the results of which are presented in this study. 
The aim of this work is to assess the impact of COVID-19 on Czech dentists.

\section{Materials and Methods}

\subsection{Design}

This ad hoc, self-administered, cross-sectional, online survey was conducted by the Czech Dental Chamber and filled out by chamber members. All participants were informed about the purpose of the study, and none of them had a patient status. The questionnaire was anonymous; reported data did not include any identifying information that could be used to trace the participants and did not allow any association with the person answering. The participants were not rewarded with any direct benefits for participating in the survey. This study was conducted in accordance with the Declaration of Helsinki.

The presented data were obtained from the answers to 9 questions. Out of these questions, 8 were close-ended, and 1 was semi-close-ended (prefilled close-ended answers along with the option to reply in an open form). The whole questionnaire was in the Czech native language and was designed in collaboration with experts from the chamber, the academic community, and general practitioners.

A description of the questions, including the type and number of answers, is given in Table 1.

Table 1. Questions and their classification.

\begin{tabular}{ccccc}
\hline Question Mark & Question & Question Type & $\begin{array}{c}\text { Number of Closed-Ended } \\
\text { Answer Options }\end{array}$ & Answer Choice \\
\hline Q1 & Sex & Closed & 2 & Single \\
Q2 & Age & Closed & 6 & Single \\
Q3 & Were you diagnosed with COVID-19 & Closed & 3 & Single \\
Q4 & by 30 June 2021? & Closed & 8 & Multiple \\
Q5 & Where did the treatment take place? & Closed & 5 & Single \\
Q6 & Do you know where you got infected? & Closed & 3 & Single \\
Q7 & Where did the transmission of & Semiclosed & 6 & Single \\
Q8 & COVID-19 occur? & Closed & 2 & Single \\
Q9 & Was another member of the team & Closed & 5 & Multiple \\
\hline
\end{tabular}

\subsection{Sample}

To address the members of the Czech Dental Chamber, invitations for participation in the survey were sent to all 9922 officially registered e-mail addresses in the chamber database. Each address represents one chamber member. The addressees were asked to fill out the questionnaire from 23 June to 4 September 2021. According to the Czech Dental Chamber 2020 Annual Report, the chamber had 11,160 members as of 31 December 2020 [14]. Thus, the survey addressed $88.9 \%$ of the chamber members. Membership in the Czech Dental Chamber is compulsory for all dentists working in the Czech Republic.

\subsection{Sample Size Relevancy}

Based on the total number of chamber members, the minimum relevant number of survey participants was set at 372 . This quantification was assessed by the online Netquest calculator using Formula (1). For the calculation, a study universe of the members of the Czech Dental Chamber $(\mathrm{N}=11,162)$, a margin of error of $5 \%$, a confidence level of $95 \%$, and a standard heterogeneity of $50 \%$ were used. As the sample size of this study (2716 participants) significantly exceeds the minimum required value $(n=372)$, the results are statistically relevant.

$$
n=\frac{\mathrm{N} \cdot \mathrm{Z}^{2} \cdot \mathrm{p} \cdot(1-\mathrm{p})}{(\mathrm{N}-1) \cdot \mathrm{e}^{2}+\mathrm{Z}^{2} \cdot \mathrm{p} \cdot(1-\mathrm{p})}
$$


Formula (1). Relevant sample size calculation. Sample size calculated (n), size of the universe $(\mathrm{N})$, deviation from the mean value $(\mathrm{Z})$, maximum margin of error tolerated $(\mathrm{e})$, expected proportion (p).

\subsection{Data Collection}

The invitation to participate was sent by e-mail to 9922 officially registered e-mail addresses of the chamber members. The e-mail contained a link to an online questionnaire in Google Forms (Google, Mountain View, CA, USA). The compatibility of the questionnaire interface was not limited and included a mobile phone, desktop computer, laptop, or tablet with support for all the most used operating systems. The collected data were stored in the Google Forms cloud database and downloaded after the whole survey was completed.

\subsection{Statistical Analysis}

After the survey was completed, the results of all the questions were downloaded from the Google Forms cloud database. The results of close-ended questions (Q1-6, Q8, and Q9) were analyzed and presented as the percentage of individual answers within all the answers provided. Blank responses were not included in the total number of responses.

Responses to the semi-close-ended question (Q7) were analyzed individually. Each open-ended answer was evaluated independently by two authors (J.S. (Jan Schmidt), V.P.). Results disagreeing between the authors were resolved by a decision of the third author (J.T.). Open responses that were of similar meaning to closed responses were transferred to the appropriate closed response category. The remaining answers were put into new groups according to their meaning. Newly formed groups that exceeded the specified limit in frequency $(n=5)$ were presented as separate answers within the results. Answers that did not exceed this limit were classified in the "Others" category. Results were analyzed and presented as the percentage of individual answers within all answers provided. Blank responses were not included in the total number of responses.

To compare the COVID-19 prevalence between the Czech Dental Chamber members and the Czech general population, it was necessary to use the same methodology. The available COVID-19 prevalence rate within the Czech general population was based on PCR-confirmed cases and did not include cases diagnosed with clinical symptoms. As of the end of this survey, the COVID-19 cumulative cases among the Czech general population was 15,546 per 100,000 people [10]. In order to compare these values with the results of our study, only PCR-verified diagnoses were used.

The data were analyzed using custom Microsoft Office Excel formulas (version 2106 for Windows, Microsoft Corporation, Redmond, WA, USA) and GraphPad Prism (version 8.0.0 for Windows, GraphPad Software, San Diego, CA, USA). Chi-square with test Yates's correction was used for statistical analysis; ${ }^{*}$ indicates $p<0.05$.

\section{Results}

\subsection{Response Rate}

A total of 2716 respondents took part in the survey. Based on the 9922 e-mails sent, the response rate was $27.4 \%$, representing $24.3 \%$ of all the chamber members $(n=11,162)$ (Figure 1). 


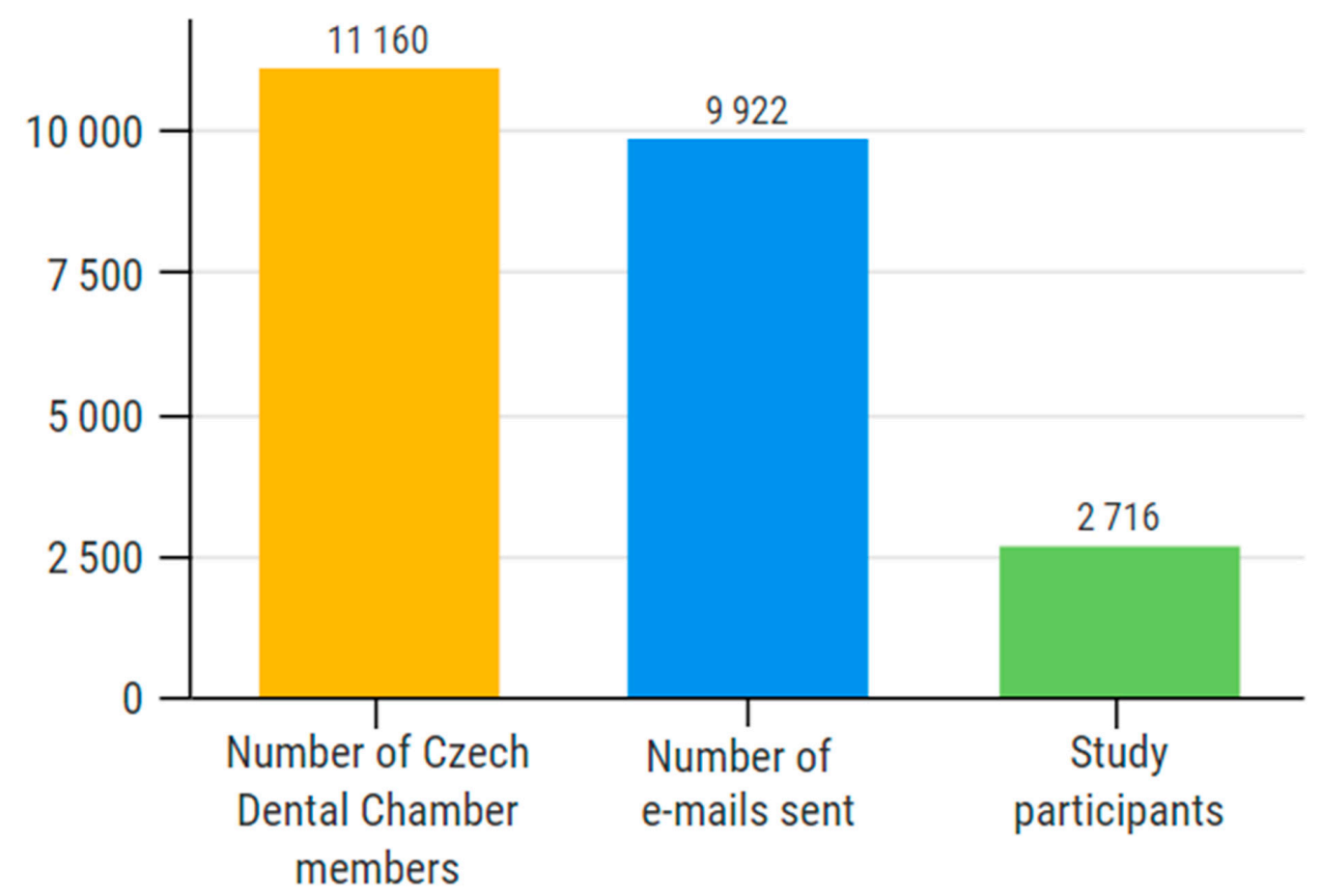

Figure 1. Response rate. The 2716 participants represent $27.4 \%$ of all the e-mail addresses included and $24.3 \%$ of the Czech Dental Chamber members.

\subsection{Sex Distribution}

A total of 2708 respondents stated their sex, and 8 skipped this question. A total of $1871(68.9 \%)$ selected the female option, and 837 (30.8\%) selected the male option (Figure 2), which also corresponds to the dominant representation of women among Czech dentists $(64.9 \%)$ [14].

\section{Sex distribution}

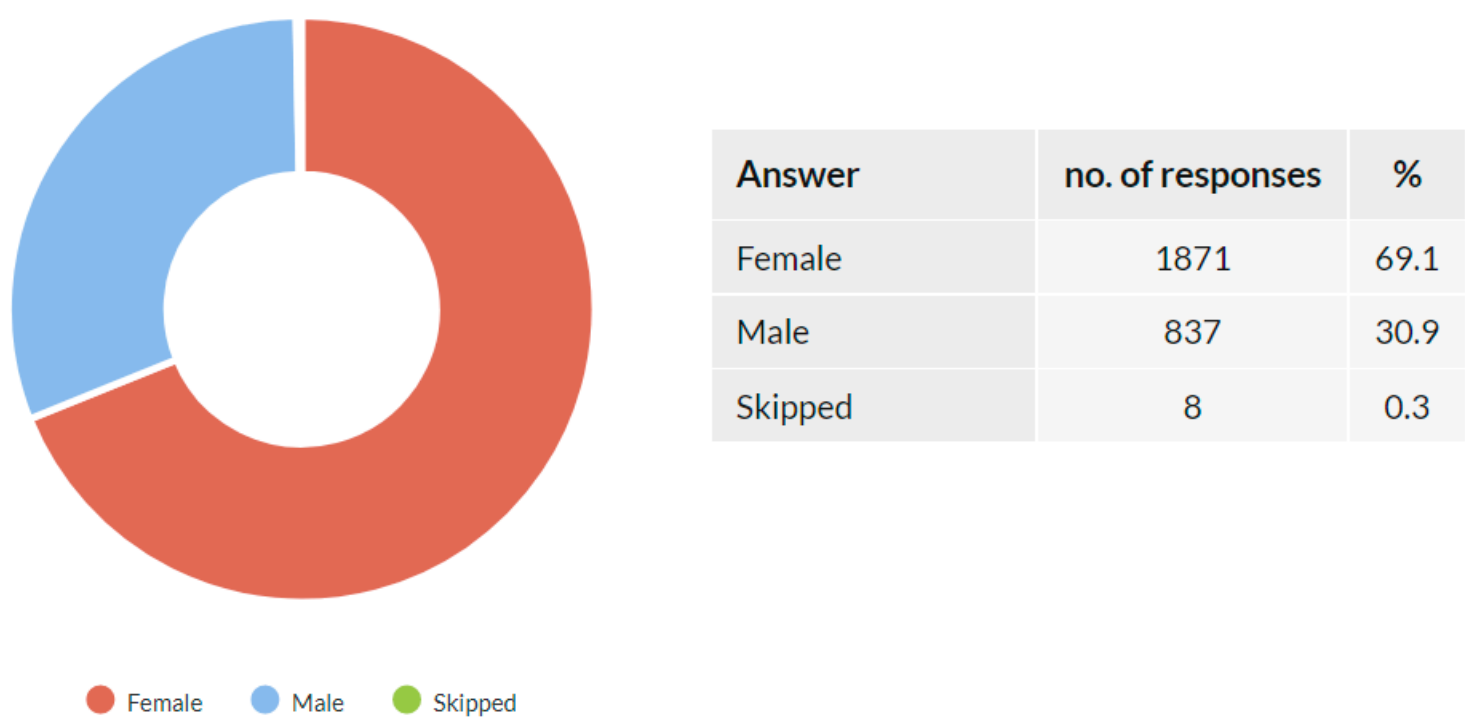

Figure 2. Sex distribution of the study participants. 


\subsection{Age Distribution}

A total of 2712 respondents stated their age, and 4 skipped this question. The distribution is illustrated in Figure 3 and approximately corresponds to the age composition of the chamber members [14]. The median age group is 50-60 years.

\section{Age distribution}

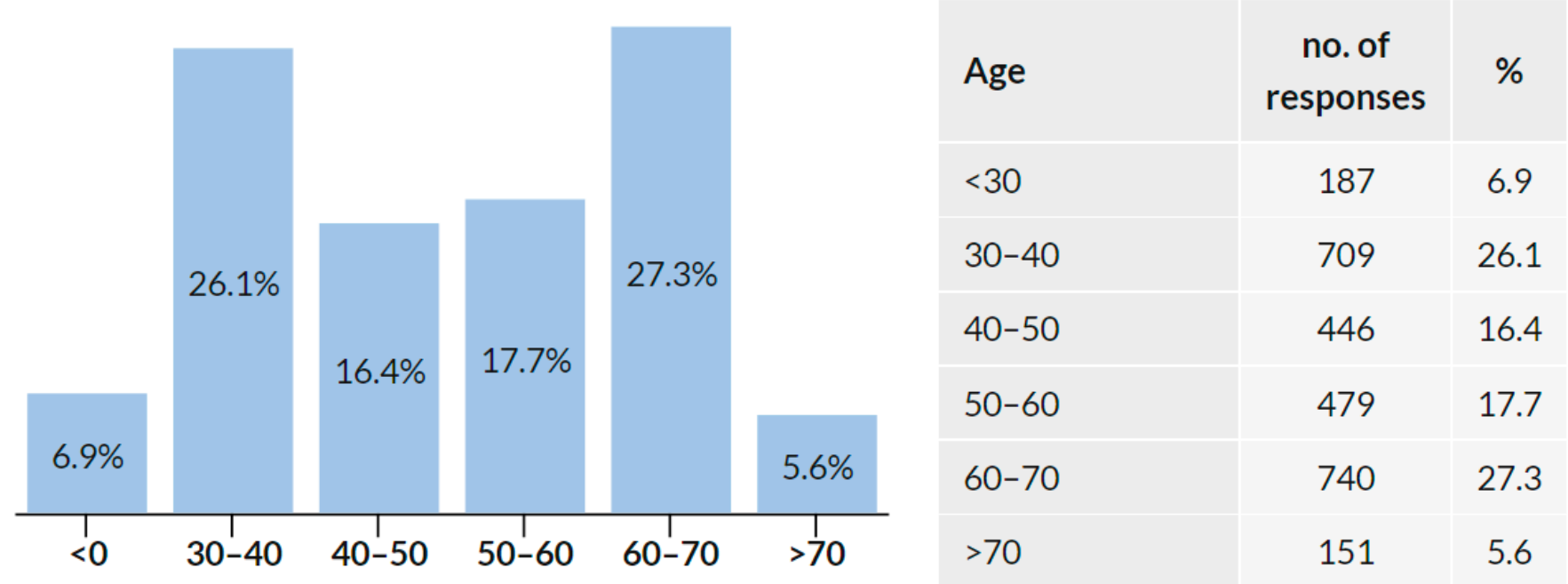

Figure 3. Age distribution of the study participants.

\subsection{COVID-19 Prevalence}

3.4.1. COVID-19 Prevalence in the Whole Study Population

A total of 2716 respondents replied to this question. No respondent skipped this question. The results are presented in Figure 4. These data reveal that $691(25.4 \%)$ respondents admitted they were diagnosed with COVID-19 by 30 June 2021.

\section{Were you diagnosed with COVID-19?}

\section{The whole study population}

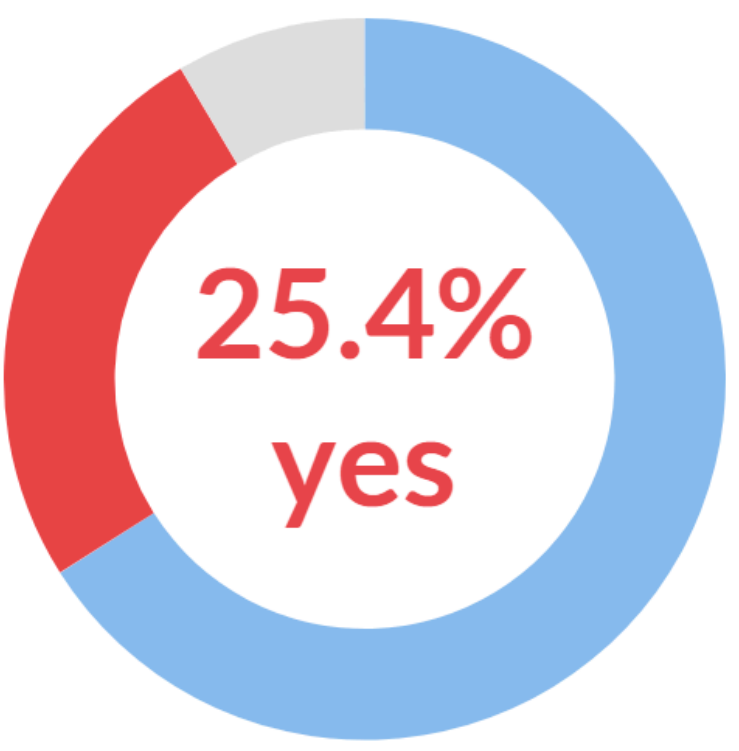

\begin{tabular}{|l|c|c|}
\hline Answer & no. of responses & $\%$ \\
\hline No & 1793 & 66.0 \\
\hline Yes & 643 & 23.7 \\
\hline Yes, only positive test & 33 & 1.2 \\
\hline Yes, repeatedly & 15 & 0.6 \\
\hline Did not know & 232 & 8.5 \\
\hline
\end{tabular}

Figure 4. COVID-19 positivity, the whole study population. 


\subsubsection{COVID-19 Prevalence Based on Sex}

Sex-based COVID-19 prevalence is provided in Figure 5. Detailed data about the answers provided are available in the Supplementary Material.

\section{Were you diagnosed with COVID-19?}

\section{Distribution by sex}

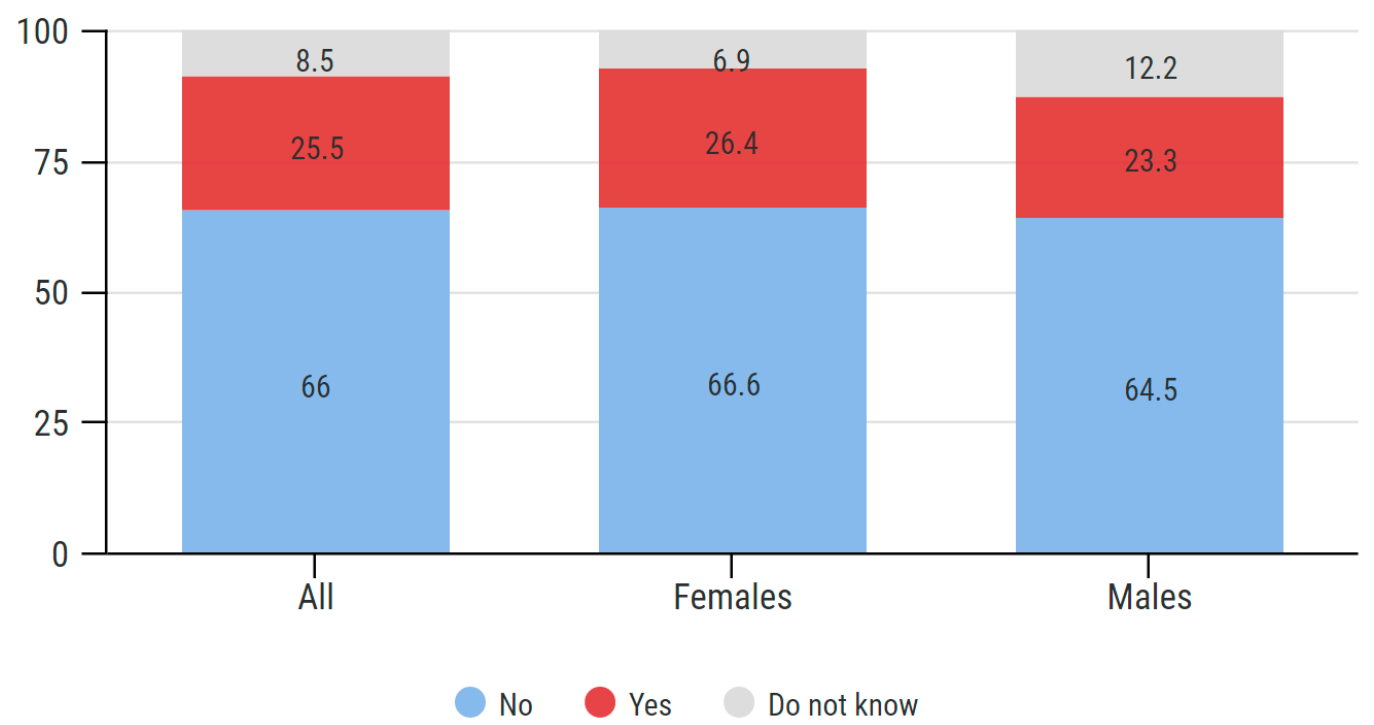

Figure 5. COVID-19 positivity, distribution by sex.

3.4.3. COVID-19 Prevalence Based on Age

Age-based COVID-19 prevalence is illustrated in Figure 6. Age- and sex-based COVID19 prevalence is shown in Figure 7. Detailed data about the answers provided are available in the Supplementary Material.

\section{Were you diagnosed with COVID-19?}

\section{Distribution by age}

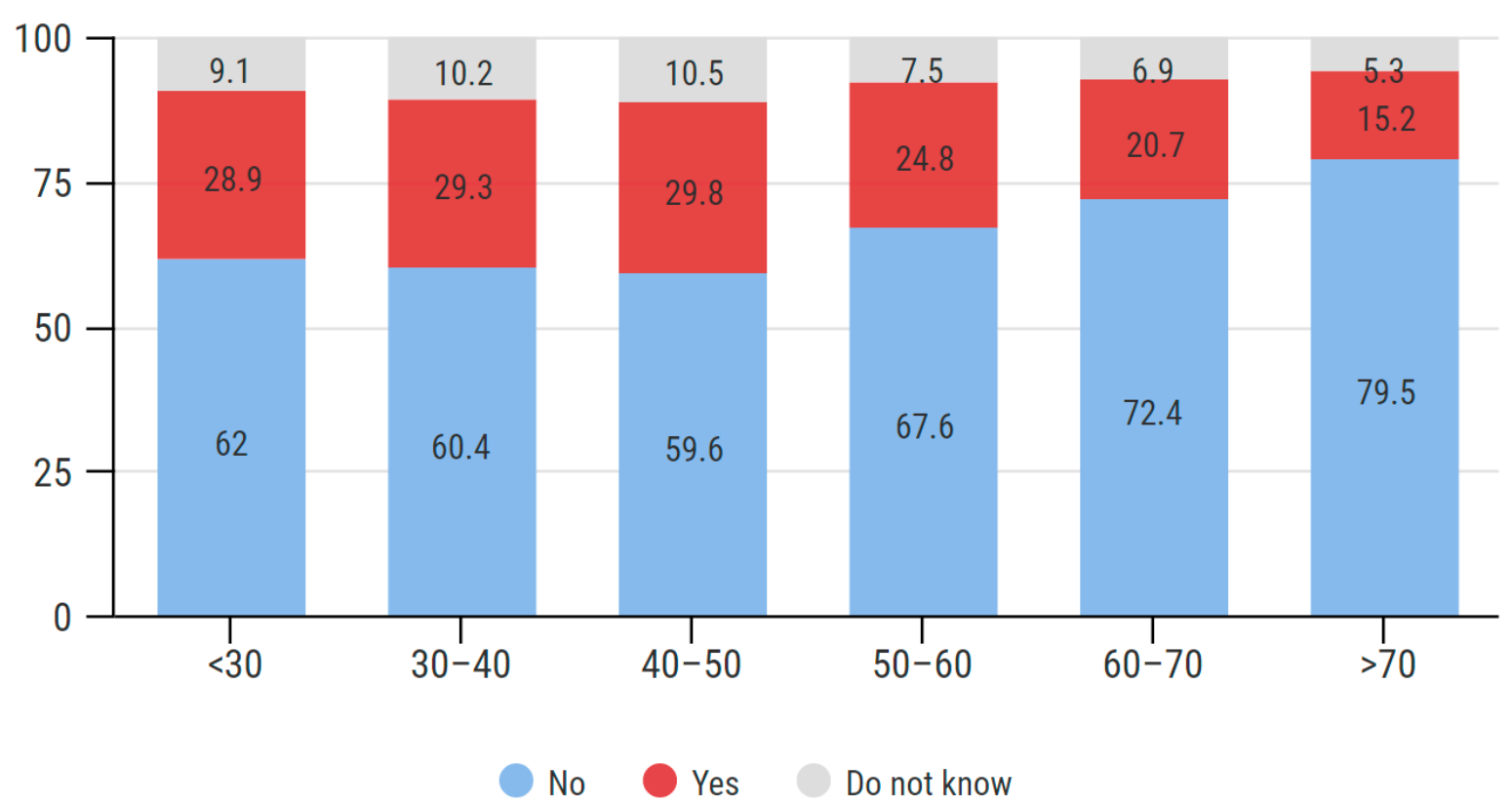

Figure 6. COVID-19 positivity, age distribution. 
Were you diagnosed with COVID-19?

$<30$

Distribution by age and sex
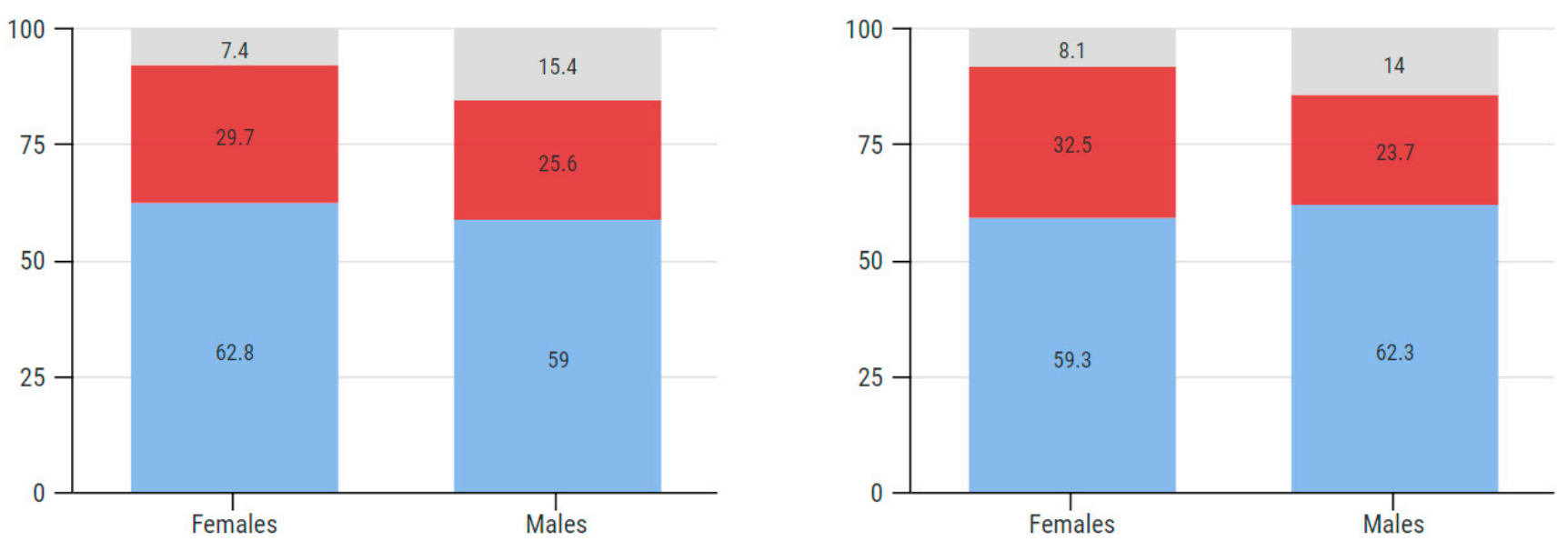

$40-50$

$50-60$
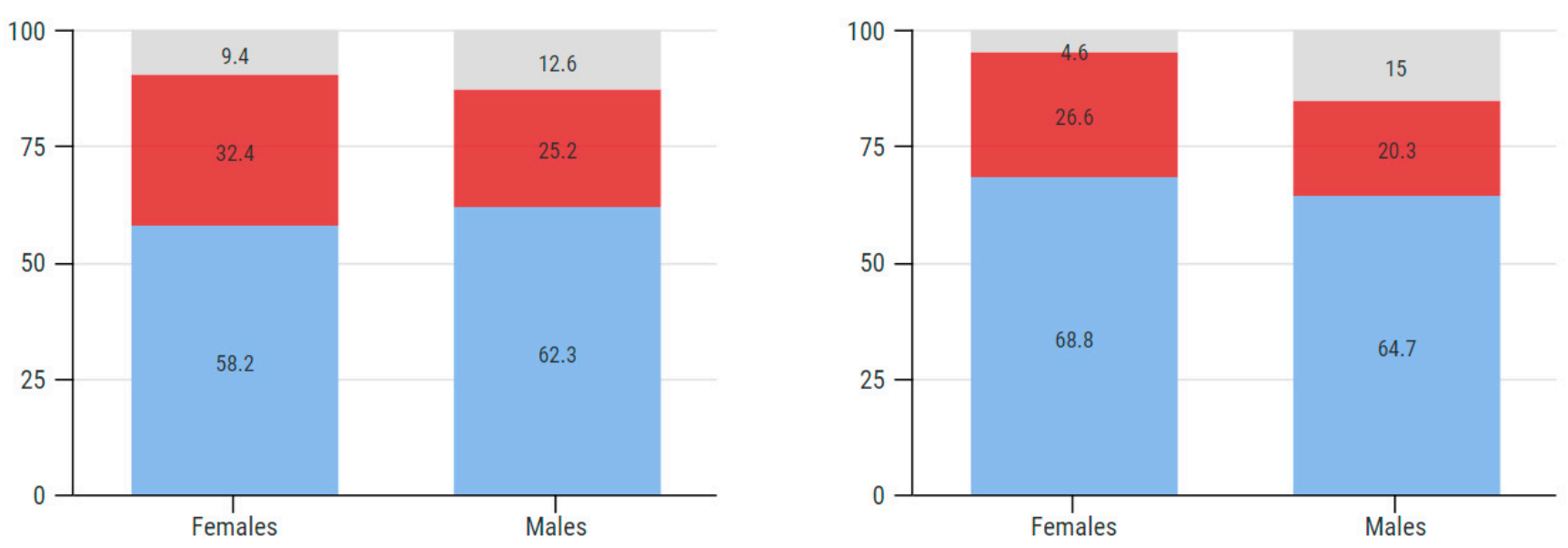

$60-70$

$>70$
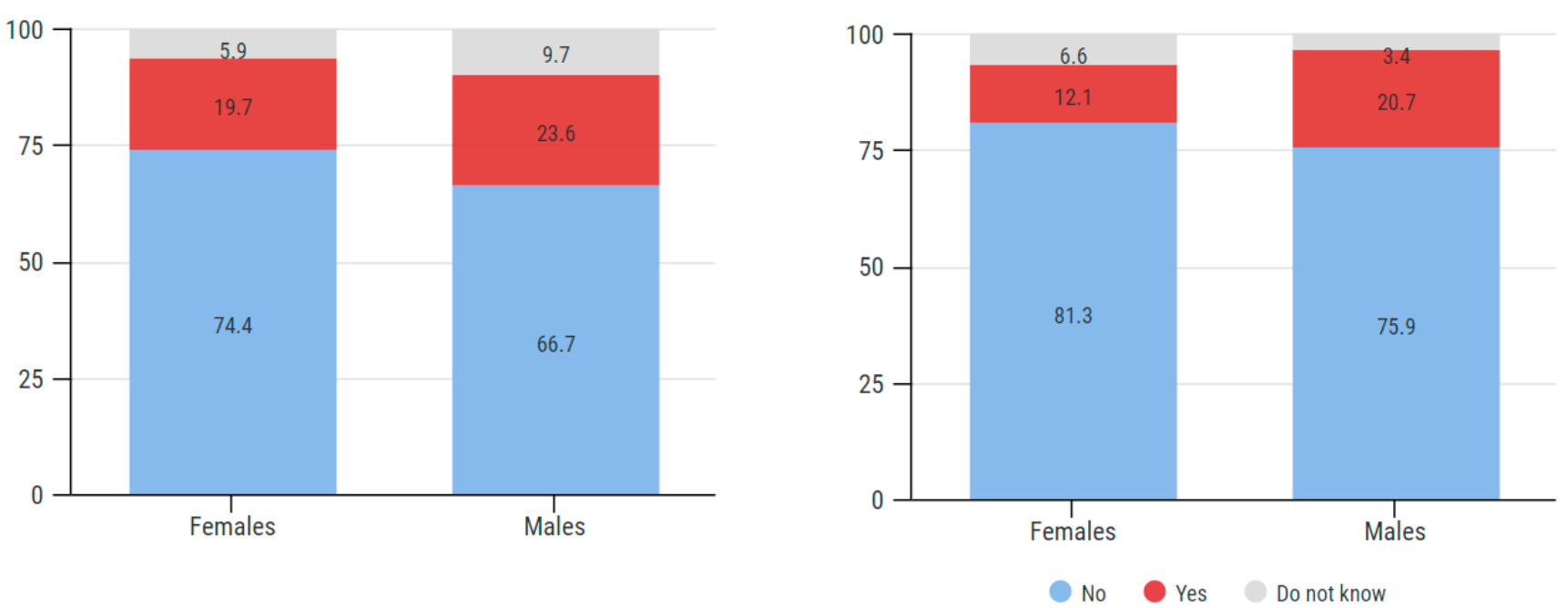

Figure 7. COVID-19 positivity, age and sex distribution. 


\subsection{COVID-19 Diagnostics}

This question was addressed only to the respondents who confirmed they were diagnosed with COVID-19 in Q3 $(n=691)$. A total of $651(94.2 \%)$ respondents reported 1328 answers to this multiple-choice question. The results are presented as a number of answers, percentage of respondents choosing this answer, and frequency of an answer among all answers in Figure 8.

\section{COVID-19 diagnostics}

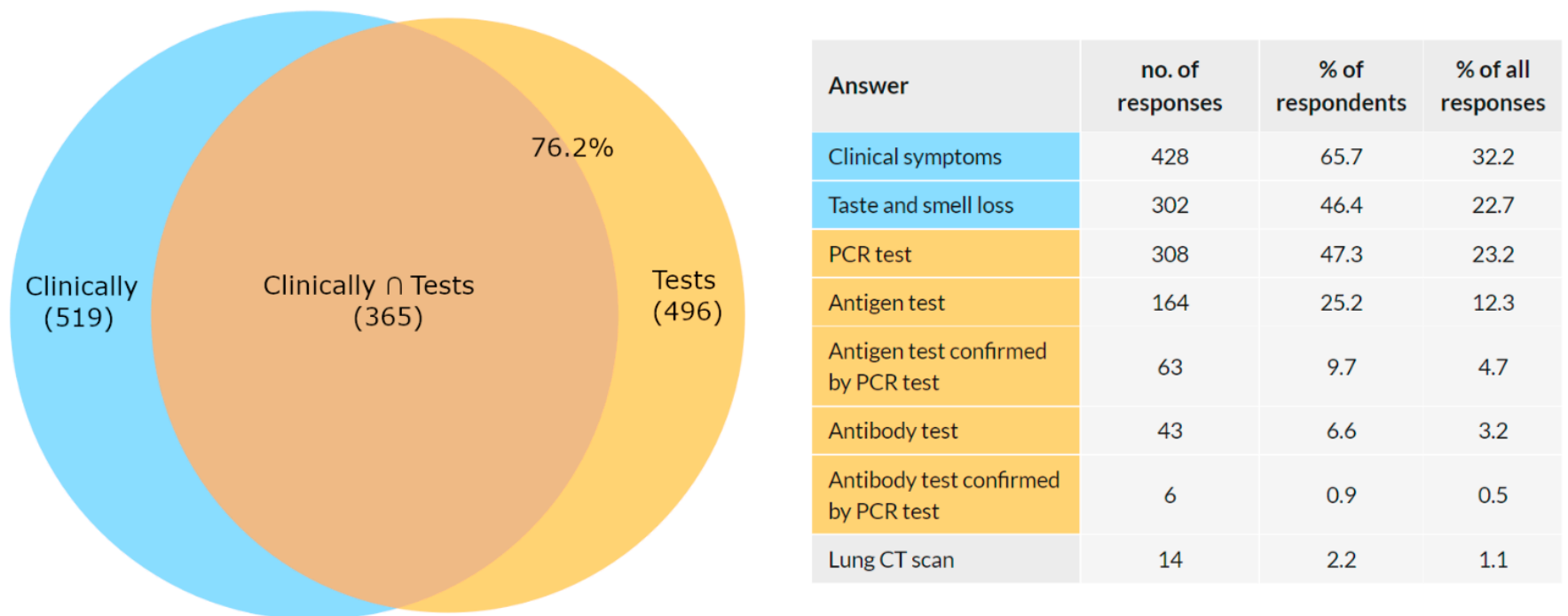

Figure 8. COVID-19 diagnostics.

There were a total of 520 respondents who chose to answer either "clinical symptoms" or "taste and smell loss". An answer containing some type of test was selected by 496 respondents. The intersection of these two groups was 365 respondents. In $76.2 \%$ of the respondents, the diagnosis of COVID-19 was confirmed by a test. In $23.7 \%$, it was diagnosed solely on the basis of clinical symptoms. In $57.9 \%$, the diagnosis was confirmed with a PCR test.

\subsection{Comparison of COVID-19 Prevalence among the General Population in the Czech Republic}

As of the end of this survey, the COVID-19 cumulative cases among the Czech general population was 15,546 per 100,000 people [10]. The PCR-verified prevalence within our study is $13.9 \%$. Compared with the PCR-verified positivity in the general population, the difference is statistically significant $(p=0.0180)$ (Figure 9 ). 


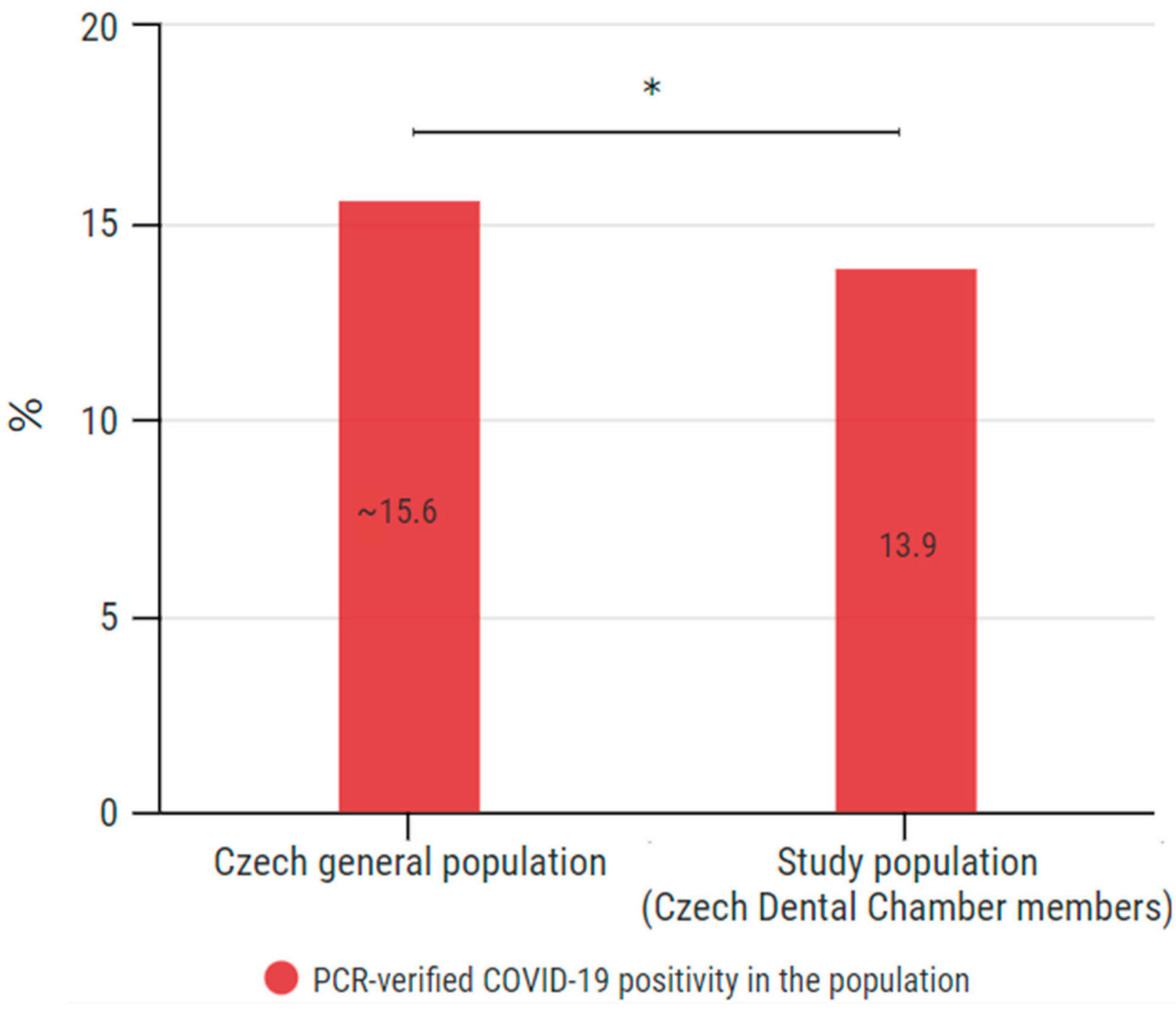

Figure 9. Comparison of COVID-19 prevalence in the Czech general population and its estimation within the population of Czech Dental Chamber members. Chi-square with test Yates's correction was used for statistical analysis; $p=0.0180$. * indicates $p<0.05$.

\subsection{Place of Treatment}

This question was addressed only to the respondents who confirmed they were infected with COVID-19 in Q3 $(n=691)$. A total of $646(93.5 \%)$ respondents answered this question.

The vast majority of the participants $(628,97.2 \%)$ answered that they were being treated in the household. Only $2.8 \%$ of COVID-19 cases led to hospitalization (Figure 10). The median age group of those hospitalized was 60-70 years. In the group of respondents older than 60 years, the infection-hospitalization ratio (IHR) was $8.7 \%$. On the other hand, none of the hospitalized were under the age of 40 ; the IHR under the age of 40 was $0 \%$. 


\section{Place of treatment}

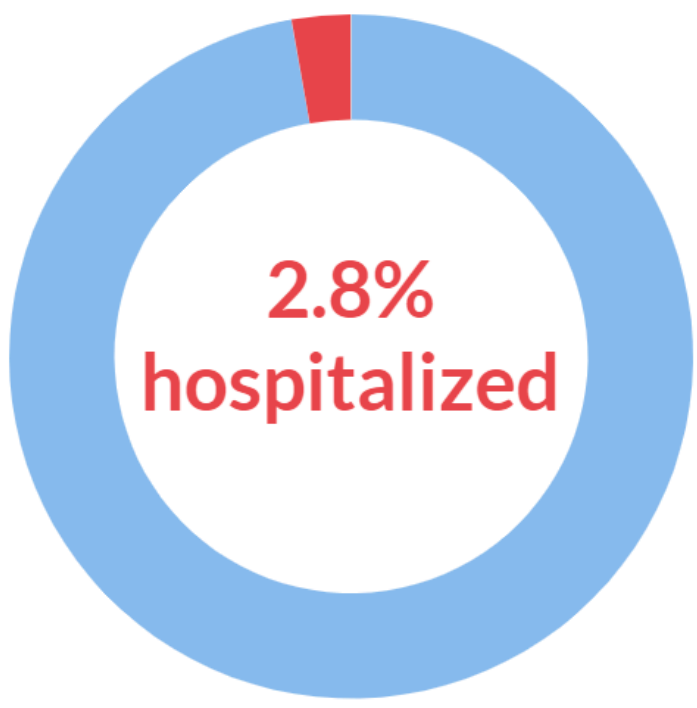

\begin{tabular}{|l|c|c|}
\hline Answer & no. of responses & $\%$ \\
\hline Household & 628 & 97.2 \\
\hline Hospital, standard & 5 & 0.8 \\
\hline Hospital, standard + O2 & 7 & 1.1 \\
\hline ICU & 4 & 0.6 \\
\hline Artificial ventilation & 2 & 0.3 \\
\hline
\end{tabular}

Figure 10. Place of treatment.

3.8. Awareness of Where the Infection Occurred

This question was addressed only to the respondents who confirmed they were diagnosed with COVID-19 in Q3 $(n=691)$. Of them, $650(94.1 \%)$ respondents answered this question. The results are provided in Figure 11.

\section{Awarness of where the infection occurred}

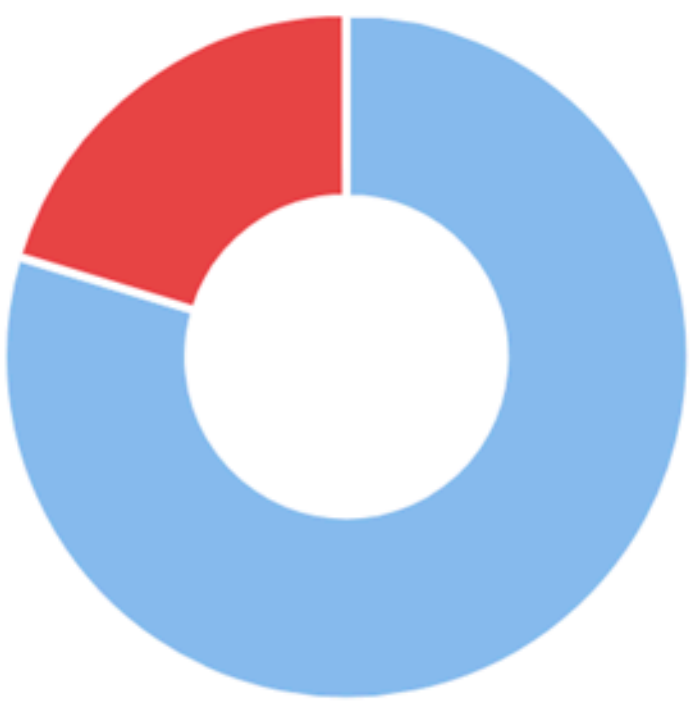

no. of responses

286

232

132

\section{$\%$}

44.0

35.7

20.3

Figure 11. Awareness of where the infection occurred.

\subsection{Environment Where the Infection Occurred}

This question was addressed only to the respondents who reported that they knew or suspected where they were infected within Q8 $(n=518)$. Of them, $517(99.8 \%)$ respondents answered this question. 
These results show that $199(38.4 \%)$ respondents identified the work environment as a source of infection. Together with the domestic environment (47.0\%), these two categories were the dominant source of infection among respondents, jointly responsible for $85.5 \%$ of the reported transmission (Figure 12). Detailed data about the answers provided are available in the Supplementary Material.

\section{Environment where the infection occurred}

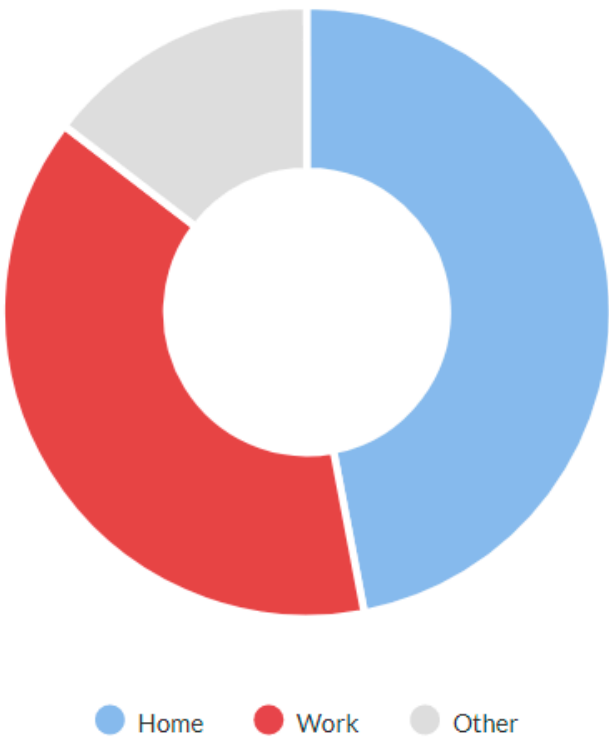

\begin{tabular}{|l|c|c|}
\hline Answer & $\begin{array}{c}\text { no. of } \\
\text { responses }\end{array}$ & $\%$ \\
\hline Household & 243 & 47.0 \\
\hline Work, via patient & 92 & 17.8 \\
\hline Work, via another team member & 53 & 10.2 \\
\hline Work, source unknown & 54 & 10.4 \\
\hline Other & 75 & 14.6 \\
\hline
\end{tabular}

Figure 12. The environment where the infection occurred.

\subsection{Prevalence of COVID-19 among Other Team Members}

This question was addressed to all survey participants. Of them, 1683 (62.3\%) replied that they were not aware of any other team member who was ill with COVID-19. A total of $1018(37.7 \%)$ respondents admitted that another team member was ill with COVID-19.

Of the respondents who admitted they were diagnosed with COVID-19, 43.3\% also reported another team member who was diagnosed as well, and 55.6\% reported that no additional team member was diagnosed. Among those respondents who replied they were not diagnosed with COVID-19,34.7\% also reported another team member who was diagnosed with COVID-19, while $65 \%$ reported that no additional team member was diagnosed with COVID-19.

\subsection{Profession Specification among Other Members of the Dental Team Infected with COVID-19}

Those who reported an additional team member diagnosed with COVID-19 in the previous question (a total of 1018 participants, 37.7\%) were asked to specify the profession of the infected individual. Of them, 990 (97.3\%) replied, providing 1124 answers to this multiple-choice question. The results are presented as a number of answers, percentage of respondents choosing this answer, and frequency of an answer among all answers in Figure 13. 


\section{Profession specification among other members of the dental team infected with COVID-19}

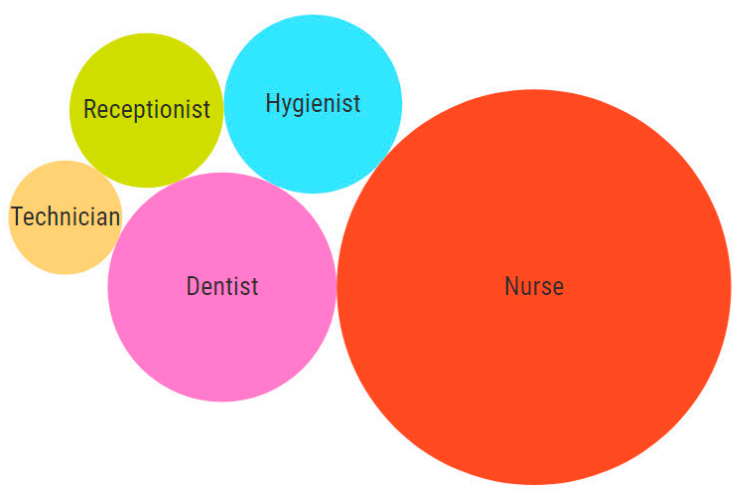

\begin{tabular}{|l|c|c|c|}
\hline Answer & $\begin{array}{c}\text { no. of } \\
\text { responses }\end{array}$ & $\begin{array}{c}\text { \% of } \\
\text { respondents }\end{array}$ & $\begin{array}{c}\text { \% of all } \\
\text { responses }\end{array}$ \\
\hline Nurse/dental assistant & 804 & 81.2 & 56.2 \\
\hline Another dentist & 271 & 27.4 & 19.0 \\
\hline Dental hygienist & 165 & 16.7 & 11.5 \\
\hline Receptionist & 123 & 12.4 & 8.6 \\
\hline Dental technician & 67 & 6.8 & 4.7 \\
\hline
\end{tabular}

Figure 13. Profession specification among other members of the dental team infected with COVID-19.

\section{Study Limitations}

There was one limitation that the authors had to address when planning this study and that they would like to discuss in this section. This limitation was not accidentally identified during the survey but was known to the authors before the research began. This chapter describes the limitation causes, possible approaches, and the approach by which the authors decided to address it.

The aim of the study is to describe the impact of COVID-19 on chamber members. In order to describe the prevalence of this disease among the respondents, it was necessary to establish diagnostic criteria. The authors considered whether these criteria would include only test-verified infections or whether they would be accepted together with diagnosis based on sole clinical symptoms.

Criteria based exclusively on tests would enhance the validity of the data. However, this method would lead to skewed results, as a large part of the Czech population was not tested and passed COVID-19 without test confirmation. At the time of the pandemic, test sites were overloaded due to the massive community-based virus spreading, and testing was unavailable to many patients. It is also important to note that one of the recommendations of the Ministry of Health of the Czech Republic was that people with COVID-19 should stay at home and be treated at home unless their condition is serious. The aim of this measure was to keep people with symptoms of COVID-19 in isolation and not to spread the infection just because of laboratory verification of the infection. Such a measure was medically correct but led to the real prevalence of COVID-19 among the population being significantly higher than the prevalence confirmed by the test.

We had two options to address this fact in determining the prevalence of COVID-19 among the study participants. One of them was to consider infected only those respondents in which positivity for COVID-19 was confirmed by a test. This option would lead to the acquisition of meticulous solid data but would significantly differ from the real prevalence. The second option was to accept the infection status regardless of the diagnostic method (i.e., both test-verified diagnosis and diagnosis based on clinical symptoms). This option would lead to less solid total data gain but would better correspond to the actual situation. In the end, we decided to obtain data combining the benefits of both of the abovementioned options.

In order to avoid skewing the results, we decided to include in the study both the group with the test-confirmed infection and the group diagnosed on the basis of clinical symptoms. To be able to distinguish these two groups in the results, the respondents were asked to indicate how COVID-19 was diagnosed, including sorting by the test used for diagnosis. Thanks to this procedure, the survey was as inclusive as possible, methodologically reflecting the epidemiological situation in the country and at the same time providing meticulous solid data. We consider this procedure to be appropriate, as it offers as much 
data as possible, within which it is still possible to sort the results on the basis of preferred criteria, such as test-verified infections.

\section{Discussion}

As there were no relevant quantitative data on the COVID-19 impact on Czech dentists, the Czech Dental Chamber decided to issue a survey among its members addressing their COVID-19 anamnesis. The data from this survey are presented in this study. Compared with studies with a similar focus and methodology, our work is one with the highest nationwide participation rates $[15,16]$.

As mentioned in the Introduction, it was assumed that the prevalence of this disease would be high in this group. This assumption was confirmed as $25.4 \%$ of the respondents stated that they were diagnosed with COVID-19. Of the total reported positive cases among the respondents, the data show that the prevalence was $26.4 \%$ among females and $23.3 \%$ among males. An interesting phenomenon was observed across age groups. While in the age groups under 50 years, the prevalence was around 30\%, with increasing age, it gradually decreased. In the group of $50-60$ years, it was $24.8 \%$, in the group of $60-70$ years $20.7 \%$, and in the group over 70 years $15.2 \%$. These results may indicate that older members of the chamber acted with more caution. It is likely that they have reduced their workload and protected themselves more. Such behavior is only logical because there is a higher risk of fatal consequences in these age groups. Overall, the highest prevalence was recorded among women aged $30-40$ and $40-50$ years (32.5\% and $32.4 \%$, respectively), and the lowest among women between $60-70$ years and above 70 years (19.7\% and $12.1 \%$, respectively). Additionally, a significant proportion (38.4\%) stated that they were infected in the work environment.

The PCR-confirmed positivity within the population of this study was $13.9 \%$. As of the end of this survey, the COVID-19 prevalence among the Czech general population was 15,546 cumulatively infected per 100,000 people ( 15.6\%) [10]. This comparison (15.6\% and $13.9 \%$ ) reveals that the prevalence among the respondents of this study was lower than in the general population. The difference is statistically significant $(p=0.0180)$. These outcomes suggest that although the dental profession is associated with a high occupational risk of droplet infection transmission, including SARS-CoV-19, the working conditions of dentists in the Czech Republic have not led to a higher prevalence of COVID-19 among them. Such results demonstrate that properly set working conditions focused on infection control led to a reduction in occupational infection risk.

For the majority of the respondents (97.2\%), COVID-19 infection did not lead to hospitalization, and they were treated at home. However, $2.8 \%$ of the participants stated that their condition required hospitalization. This result is higher than the usual rate of COVID-19-related hospitalization. However, this may be influenced by the age composition of the respondents, as the condition for entering the chamber is a university degree in dentistry. According to Manochemi et al., the COVID-19 infection-hospitalization ratio (IHR) is $2.1 \%$ [17]. However, the IHR varies considerably across age groups, ranging from $0.4 \%$ for those younger than 40 years to $9.2 \%$ for those older than 60 years. In our study, the median age of the hospitalized individuals was 60-70 years. Among those older than 60 years, the infection-hospitalization ratio (IHR) was $8.7 \%$. On the other hand, none of those hospitalized were under the age of 40 ; the IHR under the age of 40 was $0 \%$. These findings are in accordance with those of Manochemi et al.

Overall, $37.7 \%$ of the respondents admitted that another team member was diagnosed with COVID-19, of which the most frequently mentioned profession was nurse/dental assistant $(81.2 \%)$, followed by another dentist $(27.4 \%)$, dental hygienist $(16.7 \%)$, receptionist $(12.4 \%)$, and dental technician $(6.8 \%)$. These data may indicate that the distance from the site of aerosol production decreases the risk of infection. However, these results may be influenced by the uneven staffing of dental teams. Further studies would be needed to confirm this conclusion. 
To compare the prevalence of COVID-19 among Czech dentists and their foreign counterparts, it is necessary to find studies of a similar methodology carried out in a similar period of time. However, a literature search revealed a lack of studies that met both of these criteria. In June 2020, a methodologically similar work was performed by the American Dental Association [15]. The questionnaire survey addressed 2195 dentists in the USA. Of them, 355 reported that they had been tested for COVID-19. Testing via respiratory, blood, and salivary samples revealed $3.7 \%, 2.7 \%$, and $0 \%$ COVID-19 positivity. Despite the methodological similarity of this and our research, the data are not comparable, as they are separated by an interval of 1 year. Another online survey of dentists, dental hygienists, and dental assistants from around the world was conducted in August and September 2020 by Gluckman et al. [18]. The respondents were asked about the COVID-19 positivity among their dental practice staff. Of the total number of 1154 participants, $210(18.2 \%)$ admitted COVID-19 infection, of which $186(16.1 \%)$ were confirmed by a test. However, the results of this study were affected by uneven geographical participation as $48.6 \%$ of the participants were from South Africa. The COVID-19 positivity reported by the respondents from South Africa was $19 \%$, by others $13 \%$. Comparison with our study is, again, limited by the time difference of the event.

The literature search shows that studies focusing on the prevalence of COVID-19 among dentists are scant. Although many studies have been published focusing on the impact of COVID-19 on the operability of dental practices, current works on the impact of COVID-19 on dental professionals are lacking $[13,19,20]$. This condition is alarming due to the high occupational risk of dentists and emphasizes the need for further studies on this topic. Our study describing COVID-19 prevalence among members of the Czech Dental Chamber is thus one of the few that describe the impact on this professional group and, at the same time, the only one that describes this topic a year after the beginning of the pandemic.

\section{Conclusions}

This survey conducted among 2716 members of the Czech Dental Chamber reveals that $25.4 \%$ of the participants admitted to being diagnosed with COVID-19 by 30 June 2021. The total COVID-19 PCR-verified positivity was $13.9 \%$, revealing a statistically lower prevalence $(p=0.0180)$ compared with the Czech general population $(\sim 15.6 \%)$. The results of this study suggest that although the dental profession is associated with a high occupational risk of droplet infection transmission, including SARS-CoV-19, the working conditions of dentists in the Czech Republic have not led to a higher prevalence of COVID19 infection among them. Such results demonstrate that properly set working conditions focused on infection control were effective and led to a reduction in the occupational infection risk.

Supplementary Materials: The following are available online at https:/ /www.mdpi.com/article/10 .3390/ijerph182312488/s1.

Author Contributions: Conceptualization: V.P., J.S. (Jan Schmidt) and R.S.; methodology V.P. and J.T.; software: V.P. and R.S.; validation, N.P. and J.S. (Jakub Suchanek); formal analysis V.P., J.T., and J.S. (Jan Schmidt); investigation, V.P., J.S. (Jan Schmidt), J.T. and N.P.; resources, J.S. (Jakub Suchanek); data curation, V.P. and J.S. (Jan Schmidt); writing — original draft preparation, V.P., J.S. (Jan Schmidt) and J.S. (Jakub Suchanek); writing—review and editing, N.P., J.T. and R.S.; visualization, V.P. and J.S. (Jan Schmidt); supervision, J.S. (Jakub Suchanek) and R.S.; project administration, J.T., N.P. and R.S.; funding acquisition, J.S. (Jakub Suchanek) and N.P. All authors have read and agreed to the published version of the manuscript.

Funding: This work was supported by Charles University's program PROGRES Q40/13.

Institutional Review Board Statement: Not applicable.

Informed Consent Statement: Not applicable.

Data Availability Statement: The dataset is available on demand from the corresponding author. 
Acknowledgments: The authors thank Diksha Ghimire for the English language editing.

Conflicts of Interest: The authors declare no conflict of interest.

\section{References}

1. Hu, B.; Huang, S.; Yin, L. The cytokine storm and COVID-19. J. Med. Virol. 2021, 93, 250-256. [CrossRef] [PubMed]

2. Li, X.; Ma, X. Acute respiratory failure in COVID-19: Is it "typical" ARDS? Crit. Care 2020, 24, 198. [CrossRef] [PubMed]

3. Lotfi, M.; Hamblin, M.R.; Rezaei, N. COVID-19: Transmission, prevention, and potential therapeutic opportunities. Clin. Chim. Acta 2020, 508, 254-266. [CrossRef] [PubMed]

4. Goldman, E. Exaggerated risk of transmission of COVID-19 by fomites. Lancet Infect. Dis. 2020, 20, 892-893. [CrossRef]

5. Chin, A.W.H.; Chu, J.T.S.; Perera, M.R.A.; Hui, K.P.Y.; Yen, H.L.; Chan, M.C.W.; Peiris, M.; Poon, L.L.M. Stability of SARS-CoV-2 in different environmental conditions. Lancet Microbe 2020, 1, e10. [CrossRef]

6. Huang, C.; Wang, Y.; Li, X.; Ren, L.; Zhao, J.; Hu, Y.; Zhang, L.; Fan, G.; Xu, J.; Gu, X.; et al. Clinical features of patients infected with 2019 novel coronavirus in Wuhan, China. Lancet 2020, 395, 497-506. [CrossRef]

7. World Health Organization. WHO Director-General's Opening Remarks at the Media Briefing on COVID-19-11 March 2020. 2020. Available online: https:/ / www.who.int/director-general/speeches/detail/who-director-general-s-opening-remarks-atthe-media-briefing-on-covid-19---11-march-2020 (accessed on 12 July 2021).

8. Global Change Data Lab. Czechia, Coronavirus Cases per Capita, August 2020. 2021. Available online: https:/ / ourworldindata. org / explorers / coronavirus-data-explorer?tab=map\&zoomToSelection=true\&time=2020-08-31\&facet=none\&pickerSort=asc\& pickerMetric $=$ location \&hideControls=true \&Metric $=$ Confirmed + cases\&Interval $=$ Cumulative $\&$ Relative + to + Population $=$ true $\&$ Align + outbreaks $=$ false\&country $=\sim\{\}$ CZE (accessed on 10 October 2021).

9. Global Change Data Lab. Czechia, Coronavirus Deaths per Capita, August 2020. 2021. Available online: https:/ / ourworldindata. org / grapher / covid-deaths-daily-vs-total-per-million?tab=table\&time=2020-01-22..2020-08-31 (accessed on 10 October 2021).

10. Global Change Data Lab. Czechia, Coronavirus Cases per Capita, June 2021. 2021. Available online: https:/ / ourworldindata.org/ explorers / coronavirus-data-explorer?tab=table\&zoomToSelection=true\&time=earliest..2021-06-30\&facet=none\&pickerSort= asc\&pickerMetric $=$ location\&hideControls $=$ true $\&$ Metric $=$ Confirmed + cases \&Interval=Cumulative $\&$ Relative + to + Population $=$ true\&Align+outbreaks=false\&country $=\sim\{\} C Z E$ (accessed on 10 October 2021).

11. lobal Change Data Lab. Czechia, Coronavirus Deaths per Capita, June 2021. 2021. Available online: https:/ / ourworldindata.org/ grapher / total-covid-cases-deaths-per-million?tab=table\&time=2019-12-31..2021-06-30\&country= OWID_WRL (accessed on 10 October 2021).

12. Shields, A.M.; Faustini, S.E.; Kristunas, C.A.; Cook, A.M.; Backhouse, C.; Dunbar, L.; Ebanks, D.; Emmanuel, B.; Crouch, E.; Kröger, A.; et al. COVID-19: Seroprevalence and Vaccine Responses in UK Dental Care Professionals. J. Dent. Res. 2021, 100, 1220-1227. [CrossRef]

13. Schmidt, J.; Waldova, E.; Balkova, S.; Suchanek, J.; Smucler, R. Impact of COVID-19 on Czech Dentistry: A Nationwide CrossSectional Preliminary Study among Dentists in the Czech Republic. Int. J. Environ. Res. Public Health 2021, 18, 9121. [CrossRef] [PubMed]

14. Czech Dental Chamber. Ročenka ČSK 2020 (Czech Dental Chamber 2020 Annual Report). 2021. Available online: https: //www.dent.cz/o-nas/rocenky/ (accessed on 10 July 2021).

15. Estrich, C.G.; Mikkelsen, M.; Morrissey, R.; Geisinger, M.L.; Ioannidou, E.; Vujicic, M.; Araujo, M.W.B. Estimating COVID-19 prevalence and infection control practices among US dentists. J. Am. Dent. Assoc. 2020, 151, 815-824. [CrossRef] [PubMed]

16. Jungo, S.; Moreau, N.; Mazevet, M.E.; Ejeil, A.-L.; Biosse Duplan, M.; Salmon, B.; Smail-Faugeron, V. Prevalence and risk indicators of first-wave COVID-19 among oral health-care workers: A French epidemiological survey. PLoS ONE 2021, 16, e0246586. [CrossRef] [PubMed]

17. Menachemi, N.; Dixon, B.E.; Wools-Kaloustian, K.K.; Yiannoutsos, C.T.; Halverson, P.K. How Many SARS-CoV-2-Infected People Require Hospitalization? Using Random Sample Testing to Better Inform Preparedness Efforts. J. Public Health Manag. Pract. 2021, 27, 246-250. [CrossRef] [PubMed]

18. Gluckman, H.; Pontes, C.; Troelzsch, M.; Scheyer, E. An overview of COVID-19 infection in dental practices-A questionnaire survey. S. Afr. Dent. J. 2021, 76, 404-408. [CrossRef]

19. Izzetti, R.; Gennai, S.; Nisi, M.; Barone, A.; Giuca, M.R.; Gabriele, M.; Graziani, F. A perspective on dental activity during COVID-19: The Italian survey. Oral Dis. 2021, 27, 694-702. [CrossRef] [PubMed]

20. Tysiąc-Miśta, M.; Dziedzic, A. The Attitudes and Professional Approaches of Dental Practitioners during the COVID-19 Outbreak in Poland: A Cross-Sectional Survey. Int. J. Environ. Res. Public Health 2020, 17, 4703. [CrossRef] [PubMed] 